International Journal of Biology, Pharmacy and Allied Sciences (IJBPAS)

'A Bridge Betueen Caboratory and QRendo'

www.ijbpas.com

\title{
PHARMACOGNOSTICAL STUDIES AND QUALITY CONTROL PARAMETERS OF SIDA RHOMBIFOLIA
}

\section{KUMAR A ${ }^{1}$, PATHAK ${ }^{* 2}$, CHAUDHARY RP ${ }^{3}$, VERMA $V^{2}$ AND SINGH L ${ }^{4}$}

1: Department of Pharmacognosy, KSCP, Swami Vivekanand Subharti University, Meerut

$$
\text { (U.P), INDIA }
$$

2: Department of Pharmaceutical Chemistry, KSCP, Swami Vivekanand Subharti University, Meerut (U.P), INDIA

3: Department of Pharmacognosy, Umalok College of Pharmacy, Meerut, (U.P), INDIA

4: Department of Pharmacology, KSCP, Swami Vivekanand Subharti University, Meerut

$$
\text { (U.P), INDIA }
$$

*Corresponding Author: Dr. Manish Pathak: E Mail: manishpharm01@gmail.com;

Contact No.: +91-9125532749

Received 22 ${ }^{\text {nd }}$ March 2021; Revised $26^{\text {th }}$ April 2021; Accepted $3^{\text {rd }}$ June 2021; Available online $1^{\text {st }}$ Feb. 2022 https://doi.org/10.31032/IJBPAS/2022/11.2.5884

\section{ABSTRACT}

Introduction: Sida rhombifolia is a perennial plant \& grows on tropical or subtropical lands. Sida rhombifolia belongs to Malvaceae family. Sida rhombifolia has various pharmacological actions as they contain biologically active compounds. Material \& Methods: Pharmacognostical and quality control parameter involves study of macroscopic, microscopic, microchemical, TLC \& Qualitative chemical examination of Sida rhombifolia Result: All pharmacognostical and quality control parameters of Sida rhombifolia were carried out. The morphological evaluations were done to ascertain the standard reference values for standardization of the plant materials where as the microscopy, the section study of the leaves of Sida rhombifolia shows the presence of xylem, fibers, trichomes, crystal, phylum, and anisocytic stomata is present.

Keywords: Sida rhombifolia, Pharmacognostical parameters, quality control parameters 


\section{INTRODUCTION}

Sida rhombifolia (Arrow leaf Sida) is a perennial or sometimes annual plant (family-Malvaceae) native to Tropical and subtropical countries (Figure 1) [1]. Common name are huang hua mu (china), countary mallow (english), bala, mahabala (hindi), atibala (sanskrit), chittamadi (srilanka), petoria bassie (africa). These plant contains chemical constituents like Alkaloids, ascorbic-acid, beta-carotene, beta-phenethylamine, calcium, carbohydrates, ephedrine, gums, ephedrine, indole alkaloids, mucilage, phenolic compounds, protein, pseudoephedrine, saponin, steroids, tannins, triterpenoids, vascin, vasicine, flavonoids [2].

The traditional uses of Sida rhombifolia are reported like fever, ulcers, boils, urinary diseases, toothaches, relive constipation and treatment of gout etc. [3]. The pharmacological uses of Sida rhombifolia show hepatoproctive, cytotoxic, antibacterial, antifungal, anti-arthritic, antigout, anthelmintic, and hypoglycemic activities $[4,5]$.

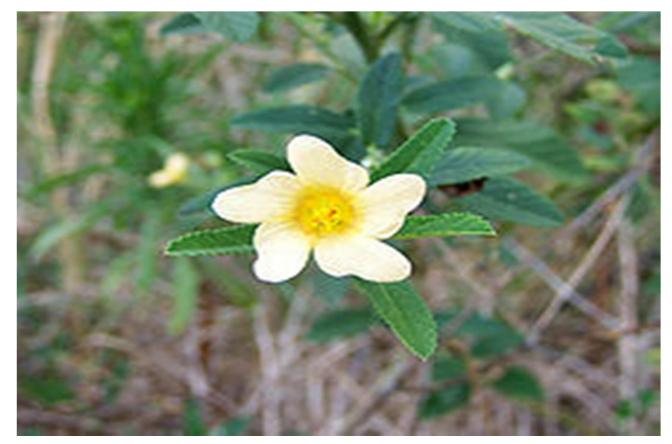

Figure 1: Flower of sida rhombifolia

\section{MATERIALS AND METHODS}

Collection and Authentication of plant material

The arial parts of sida rhombifolia $[7,8]$ were procured from F.R.I. Dehradun, Uttarakhand. The Plant material was authenticated at National Ayurveda Dietetics Research Institute (Ay), Bangalore by Dr. Shiddamallayya N (senior scientist). DrugAuthentication/SMPU/NADRI/ BAG/2011-12/540.

\section{Extraction}

Air dried powder materials of the sida rhombifolia were allowed to successive extraction with the help of soxhlet apparatus by using pet. ether, chloroform, acetone, ethanol \& water as a solvent [9].

\section{Standardization of sida rhombifolia}

\section{Macroscopic Examination}

Macroscopic parameters like size, colour, surface characteristics, texture, odour and taste was considers in above examination [10].

\section{Microchemical Tests}

Microchemical tests parameters was performed by detections of following test cellulose, chitin, suberin and cutin, starch, mucilage, protein, fixed oil and fats, tannins, calcium carbonate, inulin, cellulose cell walls, calcium oxalate [11].

\section{Microscopic Examination}

Leaf microscopy, powdered microscopy, determination of total ash, acid insoluble 
ash, water soluble ash, moisture content, swelling index, extractable matter (hot extraction \& cold extraction), foaming index, was performed in microscopic examination [12-14].

\section{Thin layer chromatography (TLC)}

Thin layer chromatography is particulary valuable for the qualitative determination of small amounts of impurities. The silica gel g slurry was made \& prepare TLC plates then allowed all plates for dry in hot air oven [14]. The mobile phase was made by the mixture of toluene: chloroform: ethanol [28.5: 57: 14.5] and all plates was stand in a suitable jar for allowed to run of mobile phase. The spots of pet.ether, chloroform, acetone, ethanol \& aqueous extracts of sida rhombifolia showed a particular chemical constituent at a particular rf value. The color of the spot visualized after apply visualizing agent like dragendorff's reagent [15-17].

Qualitative chemical examination of extracts

The following tests was performed under chemical examination of extracts-

Detection of alkaloids (mayer's test, dragendorff 's test, hager's test \& wagner's test) was performed. detection of carbohydrates (molich's test, barfoed's test). detection of glycoside (modified borntrager's test, legal's test, baljet's test), detection of saponins (foam test), detection of steroids (libermann- burchard's test), detection of fixed oils \& fats (stain test, saponification test), detection of resins (acetone-water test), detection of phenol (ferric chloride test), detection of tannin (gelatin test), detection of flavonoid (lead acetate test, zinc hydrochloric acid reduction test), detection of proteins and amino acids (millon's test, ninhydrin test, biuret test) [18, 19].

\section{RESULT AND DISCUSSION}

\section{Plant authentication}

Sida rhombifolia were authenticated at national ayurveda dietetics research institute (ay), Bangalore. (Drug authentication/smpu/nadri/bag/2011-12/540).

\section{Extraction}

The extraction was done by successive solvent extraction method using the various solvents like petroleum ether, chloroform, acetone, ethanol and aqueous solvents.

\section{Standardization}

\section{Macroscopic examination}

\section{Macroscopic examination of leaves}

The leaves are $2.5-5 \mathrm{~cm}$ long, dark green in color. The taste and odor are characteristic with obovate, acute, stellatly hair beneath surface, leaves are arranged alternately along the stem (Table 1).

\section{Macroscopic Examination of Stem}

The stems are soft and branched, growing 50 to $120 \mathrm{~cm}$ in height, with the lower sections being woody with $25-30 \mathrm{~cm}$. The colour and odour of Sda rhombifolia are 
greenish and characteristic respectively

(Table 2).

\section{Microchemical Test}

Sida rhombifolia show the presence of cellulose, lignin, fixed oils and fats, suberin and cutin, tannins, starch, mucilage, proteins, calcium oxalate crystal, cellulose cell wall, calcium carbonate, while chitin absent (Table 3).

\section{Microscopic Examination}

\section{Stomatal number and Stomatal index}

The leaves of sida rhombifolia plant contain anisocytic type of stomata. The stomatal number and stomatal index were 8 and 40 respectively. The number of epidermal cells was 12 (Table 4, Figure 1).

\section{Powder Microscopy}

Powder microscopy of sida rhombifolia was done under trinocular microscope and observed xylem, fibers, trichomes, crystal and medullary rays are present (Figure 3).

\section{Determination of total ash}

The total ash, acid insoluble ash and water soluble ash of $2 \mathrm{gm}$ of dried arieal part of sida rhombifolia were found to be $54.50 \mathrm{mg} / \mathrm{g}, \quad 32.5 \mathrm{mg} / \mathrm{g}$ and $\quad 12 \mathrm{mg} / \mathrm{g}$ respectively. The total ash value are more than acid insoluble and water soluble ash. Total ash of arial part of sida rhombifolia was found to be $54.50 \mathrm{mg} / \mathrm{g}$ (Table 5).

\section{Acid-insoluble ash}

The acid insoluble ash of sida rhombifolia was found out to be $32.5 \mathrm{mg} / \mathrm{g}$ (Table 6).

\section{Water soluble ash}

The water soluble ash of sida rhombifolia was found out to be $12 \mathrm{mg} / \mathrm{g}$ (Table 7).

\section{Determination of water matter}

Loss on drying- The water content or moisture content of aerial part of sida rhombifolia was found to be $175 \mathrm{mg} / \mathrm{g}$ (Table 8).

\section{Determination of swelling index}

The swelling index aerial part of sida rhombifolia was observed $0.25 \mathrm{ml}$ (Table 9).

\section{Determination of extractable matter}

The extractive value of sida rhombifolia in petroleum ether extract was found out to be $45 \mathrm{mg} / \mathrm{ml}$, chloroform extract $32.5 \mathrm{mg} / \mathrm{ml}$, acetone extract $47.5 \mathrm{mg} / \mathrm{ml}$, ethanol extract and in aqueous extract $65 \mathrm{mg} / \mathrm{ml}$. with cold maceration, the extractive value of petroleum ether extract was found to be $12.5 \mathrm{mg} / \mathrm{ml}$, chloroform extract 12.5 $\mathrm{mg} / \mathrm{ml}$, acetone extract $17.5 \mathrm{mg} / \mathrm{ml}$, ethanol extract $15 \mathrm{mg} / \mathrm{ml}$ and in aqueous extract it was found to be $47.5 \mathrm{mg} / \mathrm{ml}$ (Table 10).

\section{Foaming index}

The height of foam measured was less than $1 \mathrm{~cm}$ in every test tube. Therefore the foaming index was found to be less than 100 in plant (Table 11).

\section{Chromatographic studies}

The TLC of different extracts was developed with various solvent systems but the best solvent system in which spots were detected when solvent system, toluene: chloroform: ethanol (28.5: 57: 14.5) and 
detection of spots was done in dragendoff's reagent with solvent system. In pet. ether extract three spots were detected in developed TLC of sida rhombifolia whose rf value from the range of 0.47-0.84, chloroform extract showed four spots whose rf value range from 0.21-0.78, acetone extract indicated three sports with rf value from the range of $0.10-0.42$, ethanol extract of showed four sports with rf value range $0.37-0.84$ and water extract showed two spots whose rf value range from 0.15-0.21 (Table 12, Figure 4).
Qualitative chemical examination of extracts

The phytochemical screening of pet ether extract of sida rhombifolia were contains steroids, phenols and fixed oil while chloroform extract contains alkaloids and fixed oil. While acetone extract contains alkaloids, fixed oil and phenols. others ethanolic and aqueous extracts showed the presence of alkaloids, carbohydrate, protein, steroids, glycoside tannins, fixed oil, saponin, phenol, and alkaloids respectively (Table 13).

Table 1: Organoleptic characters of leaves

\begin{tabular}{|c|c|c|}
\hline S. No. & Organoleptic characters & Sida rhombifolia \\
\hline 1. & Size & $\mathbf{2 . 5 - 5 \mathrm { cm }}$ \\
\hline 2. & Surface characteristics, Texture & Obovate, Acute, Stellatly hair beneath \\
\hline 3. & Taste & Characteristics \\
\hline 4. & Color & Dark green \\
\hline 5. & Odor & Characteristic \\
\hline
\end{tabular}

Table 2: Organoleptic characters of stem

\begin{tabular}{|c|c|c|}
\hline S. No. & Organoleptic characters & Sida rhombifolia \\
\hline 1. & Size & $25-30 \mathbf{c m}$ \\
\hline 2. & Surface texture & Soft and branched \\
\hline 3. & Color & Greenish \\
\hline 4. & Odor & Characteristic \\
\hline
\end{tabular}

Table 4: Stomatal index

\begin{tabular}{|c|c|c|c|}
\hline Plant & Stomatal number $\left(\right.$ per $\left.\mathbf{m m}^{2}\right)$ & Number of epidermal cells & Stomatal index $(\mathrm{i}=\mathbf{s} / \mathbf{e}+\mathbf{s} \mathbf{x 1 0 0}) *$ \\
\hline $\begin{array}{c}\text { Sida } \\
\text { rhombifolia }\end{array}$ & 8 & 12 & 40 \\
\hline
\end{tabular}

*Where: $i=$ stomatal index, $s=$ no. of stomata per unit area, $e=$ no. of epidermal cells in the same unit area

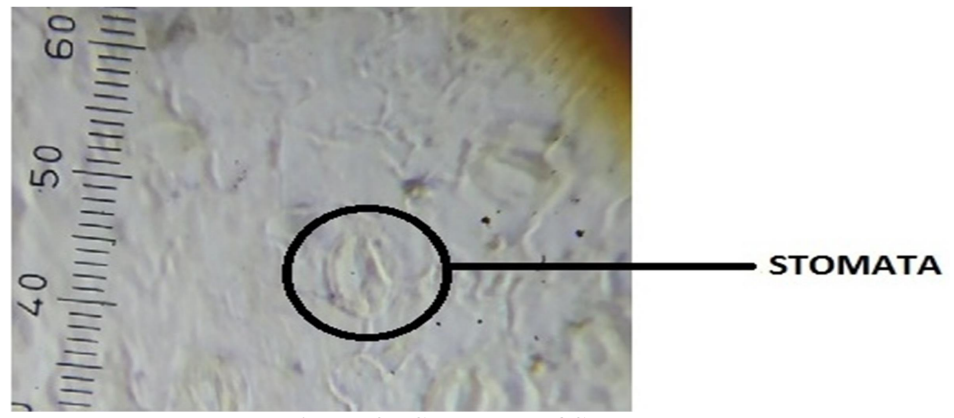

Figure 2: Structure of Stomata 
Table 3: Microchemical Test

\begin{tabular}{|c|c|c|c|c|}
\hline S. No. & Components & Test & Observation & Inference \\
\hline \multirow[b]{2}{*}{1.} & \multirow[b]{2}{*}{ Cellulose } & Iodine solution & Pale yellow & Cellulose present \\
\hline & & Iodine solution + Sulphuric acid & Bright blue & Cellulose present \\
\hline \multirow[b]{2}{*}{2.} & \multirow[b]{2}{*}{ Chitin } & Sudan red III & No red colour & Chitin absent \\
\hline & & Iodine solution + Sulphuric acid & No violet colour & Chitin absent \\
\hline \multirow{4}{*}{3.} & \multirow{4}{*}{ Lignin } & Iodine solution & Deep blue & Lignin present \\
\hline & & Iodine solution + Sulphuric acid & Brown & Lignin present \\
\hline & & Safranin & Red & Lignin present \\
\hline & & Phloroglucinol + Conc. Hcl & Stained red & $\begin{array}{l}\text { Lignin } \\
\text { Present }\end{array}$ \\
\hline 4. & Fixed oils and Fats & Chloroform & Soluble & Fixed oils \& Fats present \\
\hline \multirow{2}{*}{5.} & \multirow{2}{*}{ Suberin and Cutin } & Iodine solution & Deep yellow & Suberin and Cutin present \\
\hline & & Iodine solution + Sulphuric acid & Deep brown & Suberin and Cutin present \\
\hline 6. & Tannins & Dilute ferric chloride solution & Greenish black & Tannins present \\
\hline \multirow[b]{2}{*}{7.} & \multirow[b]{2}{*}{ Starch } & Iodine solution & Blue & Starch present \\
\hline & & Water & Blue & Starch present \\
\hline \multirow[b]{2}{*}{8.} & \multirow[b]{2}{*}{ Mucilage } & Methylene blue & Deep blue & Mucilage present \\
\hline & & Iodine solution + Sulphuric acid & Violet & Mucilage present \\
\hline \multirow{3}{*}{9.} & \multirow{3}{*}{ Proteins } & Iodine solution & Yellow crystalloids & Proteins present \\
\hline & & Alcoholic picric acid solution & Yellow crystalloids & Proteins present \\
\hline & & Millon's reagent & Brick red colour & Proteins present \\
\hline 10. & Calcium oxalate crystals & Clear the section with chloral hydrate & Insoluble in acetic acid and caustic alkali & Calcium oxalates crystals present \\
\hline 11 & Cellulose cell wall & Iodine \& Sulphuric acid solution & Blue violet & Cellulose cell wall present \\
\hline 12. & Calcium carbonate & Acetic acid & Dissolve with acetic acid & Calcium carbonate present \\
\hline
\end{tabular}




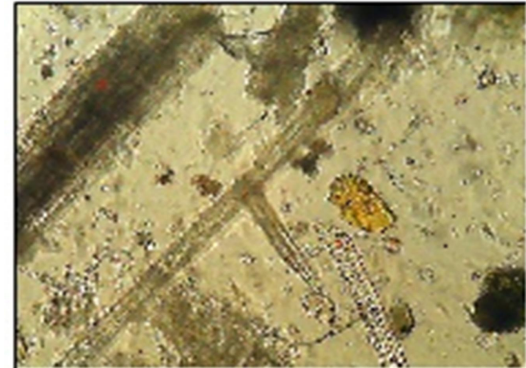

(a)

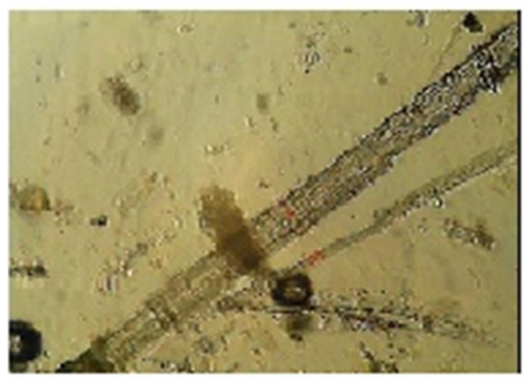

(c)

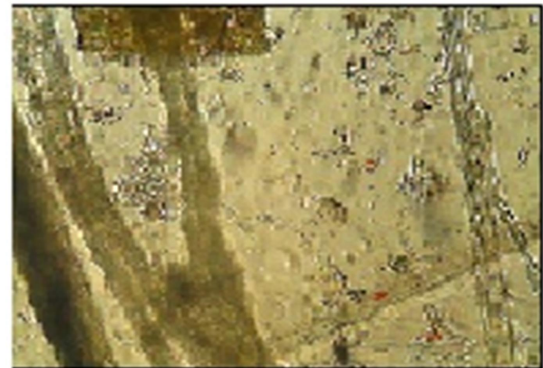

(b)

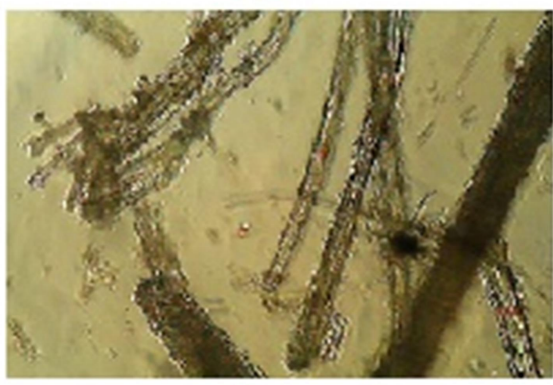

(d)

Figure 3: Powder microscopy of sida rhombifolia

Table 5: Observation of total ash

\begin{tabular}{|c|c|c|c|c|c|}
\hline Plant & $\begin{array}{c}\text { Weight of } \\
\text { crucible(g) a }\end{array}$ & $\begin{array}{c}\text { Weight of } \\
\text { drug }(\mathbf{g}) \mathbf{b}\end{array}$ & $\begin{array}{c}\text { Weight of crucible }+ \\
\text { weight of ash }(\mathrm{g}) \mathbf{c}\end{array}$ & $\begin{array}{c}\text { Ash obtained (g) } \\
(\mathbf{c}-\mathbf{a})\end{array}$ & $\begin{array}{c}\text { Total ash } \\
(\mathbf{m g} / \mathbf{g})\end{array}$ \\
\hline $\begin{array}{c}\text { Sida } \\
\text { rhombifolia }\end{array}$ & $\mathbf{2 0 . 5 5 0}$ & 2 & 21.640 & 1.09 & $\begin{array}{c}109 / 2 \\
=54.50\end{array}$ \\
\hline
\end{tabular}

Table 6: Determination of acid-insoluble ash

\begin{tabular}{|c|c|c|c|c|c|c|}
\hline Plant & $\begin{array}{l}\text { Weight of } \\
\text { crucible (g) }\end{array}$ & $\begin{array}{l}\text { Weight of } \\
\text { drug (g) }\end{array}$ & $\begin{array}{c}\text { Total } \\
\text { ash } \\
\text { (g) 'a' }\end{array}$ & $\begin{array}{c}\text { Weight of crucible }+ \\
\text { weight of acid } \\
\text { insoluble ash (g) 'b' }\end{array}$ & $\begin{array}{c}\text { Acid insoluble } \\
\text { ash obtained } \\
\text { (g) 'a-b' } \\
\end{array}$ & $\begin{array}{c}\text { Acid } \\
\text { insoluble ash } \\
(\mathrm{mg} / \mathrm{g}) \\
\end{array}$ \\
\hline $\begin{array}{c}\text { Sida } \\
\text { rhombifolia }\end{array}$ & 18.860 & 20.860 & 19.025 & 18.960 & 0.065 & 32.5 \\
\hline
\end{tabular}

Table 7: Determination of water soluble ash

\begin{tabular}{|c|c|c|c|c|c|c|}
\hline Plant & $\begin{array}{l}\text { Weight of } \\
\text { crucible (g) }\end{array}$ & $\begin{array}{l}\text { Weight of } \\
\text { drug (g) }\end{array}$ & $\begin{array}{c}\text { Total ash } \\
\text { (g) 'a' }\end{array}$ & $\begin{array}{l}\text { Weight of crucible + } \\
\text { Weight of water } \\
\text { soluble ash (g) 'b' }\end{array}$ & $\begin{array}{l}\text { Water soluble } \\
\text { ash obtained } \\
\text { (g) 'a-b' }\end{array}$ & $\begin{array}{c}\text { Water } \\
\text { soluble ash } \\
(\mathrm{mg} / \mathrm{g}) \\
\end{array}$ \\
\hline $\begin{array}{c}\text { Sida } \\
\text { rhombifolia }\end{array}$ & 21.625 & 23.625 & 21.850 & 21.825 & 0.025 & 12 \\
\hline
\end{tabular}

Table 8: Determination of water matter

\begin{tabular}{|c|c|c|c|c|c|c|}
\hline Plant & $\begin{array}{c}\text { Weight of } \\
\text { drug (g) }\end{array}$ & $\begin{array}{c}\text { Weight of } \\
\text { china dish (g) }\end{array}$ & $\begin{array}{c}\text { Total initial } \\
\text { weight (g) a }\end{array}$ & $\begin{array}{c}\text { Constant weight } \\
\text { after heating (g) b }\end{array}$ & $\begin{array}{c}\text { Difference in } \\
\text { weight (g) (a-b) }\end{array}$ & $\begin{array}{c}\text { Loss on drying } \\
(\mathbf{m g} / \mathbf{g})\end{array}$ \\
\hline $\begin{array}{c}\text { Sida } \\
\text { rhombifolia }\end{array}$ & 4 & 65.60 & 69.60 & 68.90 & 0.7 & $\begin{array}{c}700 / 4 \\
=175\end{array}$ \\
\hline
\end{tabular}

Table 9: Swelling index

\begin{tabular}{|c|c|c|c|c|c|c|c|}
\hline \multirow[t]{2}{*}{ S. No. } & \multirow[t]{2}{*}{ Time } & \multicolumn{3}{|c|}{$\begin{array}{l}\begin{array}{l}\text { Readings of sida } \\
\text { rhombifolia }(\mathrm{ml})\end{array} \\
\end{array}$} & \multirow{2}{*}{$\begin{array}{c}\begin{array}{c}\text { Mean } \\
(\mathrm{ml})\end{array} \\
\mathrm{m}_{1} \\
\end{array}$} & $\begin{array}{c}\text { Differ. for 4g plant } \\
\text { material } d=(b-a)(\mathrm{ml})\end{array}$ & $\begin{array}{c}\text { Swelling index for } 1 \mathrm{~g} \\
(\mathrm{~d} / 4)(\mathrm{ml})\end{array}$ \\
\hline & & $\mathbf{r}_{1}$ & $\mathbf{r}_{2}$ & $\mathbf{r}_{3}$ & & \multirow[b]{3}{*}{1} & \multirow[b]{3}{*}{0.25} \\
\hline 1 & Initially & 25 & 25 & 25 & 25 (a) & & \\
\hline 2 & $\begin{array}{c}\text { After } \\
\text { 3hours }\end{array}$ & 26 & 26 & 26 & 26 (b) & & \\
\hline
\end{tabular}


Table 10: Swelling index

\begin{tabular}{|c|c|c|c|c|c|c|c|}
\hline Method & $\begin{array}{l}\text { Dru } \\
\text { g (g) }\end{array}$ & Extract & $\begin{array}{l}\text { Wt. of } \\
\text { empty china } \\
\operatorname{dish}(g)\end{array}$ & $\begin{array}{c}\text { Wt. of china dish } \\
+ \text { dried extract } \\
\text { (g) }\end{array}$ & $\begin{array}{c}\text { Difference } \\
\text { (mg) }\end{array}$ & $\begin{array}{c}\text { Extractable } \\
\text { matter } \\
(\mathrm{mg} / \mathrm{g})\end{array}$ & Color of extract \\
\hline \multirow{5}{*}{ Hot } & 4 & Pet. Ether & 50.28 & 50.46 & 180 & 45 & Reddish Brown \\
\hline & 4 & Chloroform & 48.43 & 48.56 & 130 & 32.5 & Brownish \\
\hline & 4 & Acetone & 53.04 & 53.23 & 190 & 47.5 & Reddish brown \\
\hline & 4 & Ethanol & 50.75 & 50.76 & 10 & 2.5 & Reddish brown \\
\hline & 4 & Aqueous & 53.90 & 54.16 & 260 & 65 & Reddish brown \\
\hline \multirow{5}{*}{ Cold } & 4 & Pet. Ether & 50.13 & 50.18 & 50 & 12.5 & Yellow \\
\hline & 4 & Chloroform & 48.29 & 48.34 & 50 & 12.5 & Yellowish green \\
\hline & 4 & Acetone & 52.94 & 53.01 & 70 & 17.5 & Greenish \\
\hline & 4 & Ethanol & 50.40 & 50.46 & 60 & 15 & Light Green \\
\hline & 4 & Aqueous & 53.66 & 53.85 & 190 & 47.5 & Reddish brown \\
\hline
\end{tabular}

Table 11: Observation of height of foam

\begin{tabular}{|c|c|c|c|c|c|c|c|c|c|c|}
\hline Test tube & 1 & 2 & 3 & 4 & 5 & 6 & 7 & 8 & 9 & 10 \\
\hline Ratio of water: extract & $9: 1$ & $8: 2$ & $7: 3$ & $6: 4$ & $5: 5$ & $4: 6$ & $3: 7$ & $2: 8$ & $1: 9$ & 0:10 \\
\hline Height of foam in sida rhombifolia $(\mathrm{cm})$ & $\mathbf{0}$ & $\mathbf{0}$ & $\mathbf{0}$ & $\mathbf{0}$ & $\mathbf{0}$ & $\mathbf{0}$ & $\mathbf{0}$ & 0.2 & 0.2 & 0.3 \\
\hline
\end{tabular}

Table 12: TLC with solvent system toluene: chloroform: ethanol (28.5:57:14.5)

\begin{tabular}{|c|c|c|c|c|c|c|}
\hline S. No. & Plant & Extract & $\begin{array}{c}\text { Distance } \\
\text { travelled by } \\
\text { solute }(\mathrm{cm})\end{array}$ & $\begin{array}{c}\text { Distance } \\
\text { travelled by } \\
\text { solvent }(\mathrm{cm})\end{array}$ & $\mathbf{r}_{\mathrm{f}}$ value & Color of spot \\
\hline \multirow[t]{3}{*}{1} & \multirow{3}{*}{ Sida rhombifolia } & \multirow{3}{*}{ Petroleum ether } & \multirow{3}{*}{9.5} & 4.5 & 0.47 & Green \\
\hline & & & & 7.5 & 0.78 & Green \\
\hline & & & & 8.0 & 0.84 & Light green \\
\hline \multirow{4}{*}{2.} & \multirow{4}{*}{ sida rhombifolia } & \multirow{4}{*}{ Chloroform } & \multirow{4}{*}{9.5} & 2.0 & 0.21 & Yellow \\
\hline & & & & 4.5 & 0.47 & Light yellow \\
\hline & & & & 6.5 & 0.68 & Light green \\
\hline & & & & 5.0 & 0.78 & Yellow \\
\hline \multirow{3}{*}{3.} & \multirow{3}{*}{ sida rhombifolia } & \multirow{3}{*}{ Acetone } & \multirow{3}{*}{9.5} & 1.0 & 0.10 & Light yellow \\
\hline & & & & 1.5 & 0.15 & Green \\
\hline & & & & 40 & 0.42 & Light green \\
\hline \multirow{4}{*}{4.} & \multirow{4}{*}{ sida rhombifolia } & \multirow{4}{*}{ Ethanol } & \multirow{4}{*}{9.5} & 3.6 & 0.37 & Yellow \\
\hline & & & & 5.0 & 0.47 & Light yellow \\
\hline & & & & 6.5 & 0.68 & Green \\
\hline & & & & 4.5 & 0.84 & Light green \\
\hline \multirow{2}{*}{5.} & \multirow{2}{*}{ sida rhombifolia } & \multirow{2}{*}{ Water } & \multirow{2}{*}{9.5} & 1.5 & 0.15 & Light yellow \\
\hline & & & & 2.0 & 0.21 & Green \\
\hline
\end{tabular}

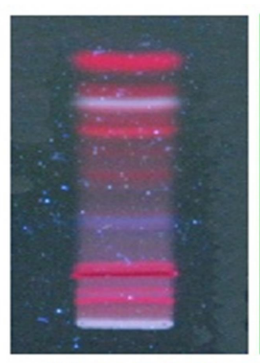

(a) TLC of PE SR

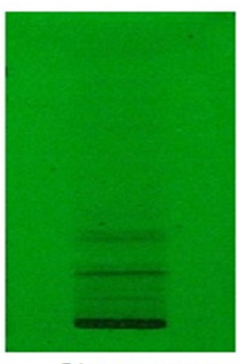

(b) TLC of CESR

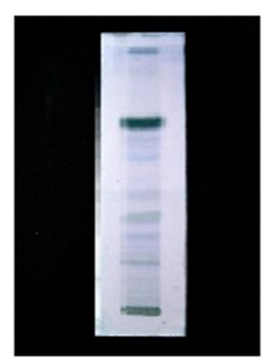

(c) TLC of AESR

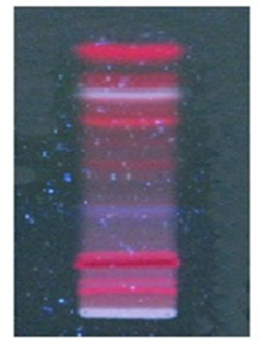

(d) TLC of SSR

Figure 4: TLC

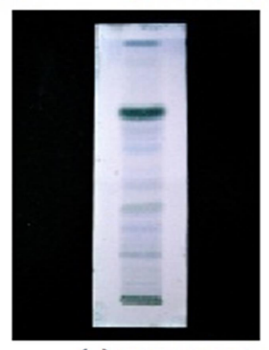

(e) TLC of AESR

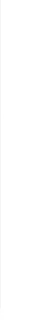


Table 13: Inferences of qualitative chemical examination of extracts

\begin{tabular}{|c|c|c|c|c|c|c|c|}
\hline \multirow{2}{*}{ S. No } & \multirow{2}{*}{$\begin{array}{c}\text { Chemical } \\
\text { constituents }\end{array}$} & \multirow{2}{*}{ Test } & \multicolumn{5}{|c|}{ Sida rhombifolia extracts } \\
\hline & & & Pet. ether & Chlorofom & Acetone & Ethanol & Aq. \\
\hline \multirow{4}{*}{1.} & \multirow{4}{*}{ Alkaloids } & Mayers test & - & + & + & + & + \\
\hline & & Dragendorff's test & - & + & + & + & - \\
\hline & & Wagners test & - & + & + & + & - \\
\hline & & Hagers test & - & + & - & + & - \\
\hline \multirow{3}{*}{2.} & \multirow{3}{*}{ Carbohydrates } & Molisch's test & - & - & - & + & - \\
\hline & & Benedict's test & - & - & - & + & - \\
\hline & & Fehling's test & - & - & - & + & - \\
\hline \multirow{2}{*}{3.} & \multirow{2}{*}{ Glycosides } & Modified borntragers & - & - & - & + & - \\
\hline & & Legal test & - & - & - & + & - \\
\hline \multirow{3}{*}{4.} & \multirow{3}{*}{ Steroids } & Salkowski's test & - & - & - & + & - \\
\hline & & Sulfur powder test & + & - & - & + & - \\
\hline & & Libermann burchard & + & - & - & - & - \\
\hline 5. & Resins & Acetone-water test & - & - & - & - & - \\
\hline 6. & Phenols & Ferric chloride test & + & - & + & + & - \\
\hline 7. & Tannins & Gelatin test & - & - & - & + & - \\
\hline \multirow{3}{*}{8.} & \multirow{3}{*}{ Flavonoids } & Alk. reagent test & - & - & - & - & - \\
\hline & & Zn-hcl acid reduction & - & - & - & - & - \\
\hline & & Shinoda test & - & - & - & - & - \\
\hline 9. & Fixed oils & Filter paper & + & + & + & - & - \\
\hline \multirow{3}{*}{10} & \multirow{3}{*}{ Protenis } & Millons test & - & - & - & - & - \\
\hline & & Ninhydrine test & - & - & - & + & - \\
\hline & & Biuret test & - & - & - & + & - \\
\hline
\end{tabular}

\section{CONCLUSION}

In this present study, Pharmacognostical and quality control parameters of Sida rhombifolia were studied. The morphological evaluations were done to ascertain the standard reference values for standardization of the plant materials, in which sida rhombifolia considers the parameters like macroscopic examination of leaves and stem, microchemical tests, stomatal number and stomatal index, powder microscopy, determination of total ash (acid insoluble and water soluble), loss of drying, swelling index, foaming index, determination of extractable matter, chromatographic studies, qualitative chemical examination. In the microscopy, the section study of the leaves of Sida rhombifolia shows the presence of xylem, fibers, trichomes, crystal, phylum, and anisocytic stomata is present.

\section{FUNDING}

Self financed. Not funded by any agency of country or outside the country.

\section{ACKNOWLEDGEMENTS}

Authors are sincerely thankful to National Ayurveda Dietetics Research Institute (Ay), Bangalore for authentication of plant, SAIF, Punjab University (Punjab) for characterization work \& Kharvel Subharti College of Pharmacy, Swami Vivekanand Subharti University, Meerut (U. P.) for providing research facilities.

\section{AUTHOR CONTRIBUTIONS}

Conduct of the study, data collection, analysis and interpretation (Kumar Amit, Pathak Manish); Sample characterization (Chaudhary Ramesh); Sample testing 
(Verma Vikrant); Manuscript preparation, design and review (Kumar Amit, Pathak M., Singh Lubhan)

\section{REFERENCES}

[1] Alam M, Saraswathy $\mathrm{V}$ N, Venugopalan $\mathrm{T} \quad \mathrm{N}$, Jaya $\mathrm{N}$ Namboodiri. Standardization studies on Ksirabala tailam. Aryavaidyam, 1998; 3:11: $164-167$.

[2] Asha Sankar M, Nair G S, Augustine A. Ephedrine synthesis in in vitro cultures of Sida through precursor feeding. Journal of Medical and Aromatic Plant Sciences, 2000; 1: 22: 65.

[3] Anil Kumar, Aditya P Yadav, D K. Medico - ethanobotanical study Oraon and Munda tribals of Chotanagpur Bihar Proceeding of the 88th Indian Science Congress, 2002; 4: 3: 63 .

[4] Ashok P, Arulmozhi S, bhaskara B P, Rajendran R. Comparative food intake inhibitory activity if Sida cordifolia L. and Withania somnifera L. in rats. Journal of Natural Remedies, 2007; 2: 7: 289 293.

[5] Akhtar M S, Iqbal J. Evaluation of the hypoglycemic effect of Achyranthes aspera in normal and alloxan diabetic rabbits. J.Ethnopharmacol.2009; 1: 31: 260 -265 .
[6] Babu V, Gangadevi T, Subramanian A. Antidiabetic activity of ethanol extract of cassia kleinii leaf in streptozotocin - induced diabetic rats and isolation of an active fraction and toxicity evaluation of the extract. Indian Journal of Pharmacology; 2013: 35: 290 - 296.

[7] Banzouzi J T, Prado R, Menan H, Vlentin A, Roumestan C, Mallie, M. Stuies on medicinal plants of ivory coast, investigation of Sida acuta for in vitro antiplasmodial activities and identification of an active constituent. Phytomedicine; 2004: 4: 11(4): 338 - 341.

[8] Balakrishnan N, Balasubramanian A, Jeyakandan M, Bhaskar V H, Sangameswaran B. Pharmacognostical and Phytochemical Evaluation of Sida cordifolia L. root. Jona of Arc, J; 2007: 1: 7: 157 - 159 .

[9] Bharat Gami, Parabia M H. Pharmacognostic Evaluation of bark and seeds of Mimusops elengi L. Int. Journal of Pharmacy and Pharmaceutical sciences; 2010: 4: 2: 123- 128

[10] Biren N Shah, Jalalpure $\mathrm{S} S$, Nayak B S. Text book of Pharmacognosy and Phytochemistry. Elsevier Health Sciences; 2009: 2: 256. 
[11] Bottini N, Vang T, Cucca F, Mustelin T. Role of PTPN22 in type 1 diabetes and other autoimmune diseases. Seminars in Immunol; 2006: 18: 207 - 213.

[12] Chatterjee S, Pramanick N, Chattopadhyay S, Munian K, Kolhapure S A, Evaluation of the efficacy and safety of nourishing baby oil in infantial xerosis. Antiseptic; 2005: 4: 102: 179 182.

[13] Charles B, Heiser. Weeds in my garden, observation on some misunderstood plants. Timber press; 2007: $643-644$.

[14] Coll J, Reixach N, Sanchez Baeza F, Casas J, Camps F. New ecdysteroids from Polypodium vulgare. Tetrahedron; 1994: 24: 50: $7247-7252$.

[15] Dubey GP, Dixit SP, Alok S. Alloxaninduced diabetes in rabbits and effect of herbal formulation D-400. Indian J. Pharmacol; 1994: 26: 225 - 236.

[16] Ekramul Islam M, Ekramul Haque M, Mosaddik M A. Cytotoxicity and antibacterial activity of Sida rhombifolia grown in Bangladesh. Phytotherapy research; 2002: 8: $17: 973-975$
[17] Egonsthal, Thin Layer Chromatography Springer; 2005: 105

[18] Edeoga H O, Okwu D E, Mbaebie B O. Minerals and Nutritive value of some Nigerian medicinal plant. Journal of Medical and Aromatic Plant Sciences; 2003: 4: 1010 1015.

[19] Faten MM Darwish, Manfred G Reinecke. Ecdysteroids and other constituents from Sida spinosa L. Phytochemistry; 2003: 62: 1179 1184. 